\title{
Métodos de producción en el cultivo intensivo de camarón blanco (litopenaeus vannamei) en baja salinidad, una opción para familias emprendedoras
}

\section{Production methods in the intensive cultivation of white shrimp (litopenaeus vannamei) in low salinity, an option for enterprising families}

MSc. Dalton Ignacio Aguirre Celi ${ }^{1}$ daltonaquirre@hotmail.com

PhD. Milton Rafael Maridueña Arroyave ${ }^{2}$ milton.mariduenaa@ug.edu.ec

PhD. Carlos Ching ${ }^{3}$

MSc. Oscar Pérez Perdomo 4 operezp74@gmail.com

Recibido: 1/04/2019; Aceptado: 1/06/2019

\begin{abstract}
RESUMEN
El estudio de los diferentes métodos de cultivo de camarón blanco (Litopenaeus vannamei) en aguas de baja salinidad, ya sean estas de pozos subterráneos o de estuarios, es evaluado el desarrollo desde la recepción de la larva en un tamaño de 350 animales por gramo aclimatada a salinidades bajas, mediante un proceso de aclimatación en campo colocándolas por un lapso de 10 días en raceways o estanques pequeños de 100 toneladas de agua, va permitir dar un mejor desarrollo y manejo cuidando su sobrevivencia. Luego se hace la transferencia a las pre crías o piscinas de pre engorde permaneciendo unos 25 días donde alcanzan un peso adecuado siendo estos juveniles para su posterior siembra en las piscinas de engorde final donde se busca alcanzar en el menor tiempo posible una talla adecuada para comercializar y manteniendo las sobrevivencias hasta el final del ciclo. Esto se realizó en Ecuador, provincia El Oro, cantón Huaquillas.
\end{abstract}

Palabras Clave: camarón, salinidad, días de cultivo, sobrevivencia

\footnotetext{
1 Administrador de MIAGROCAM, Guayaquil, Ecuador.

2 Universidad de Guayaquil, Guayaquil, Ecuador.

3 Gerente de Acuicultura Vitapro S.A, Guayaquil, Ecuador.

4 Vitapro S.A, Guayaquil, Ecuador.
} 


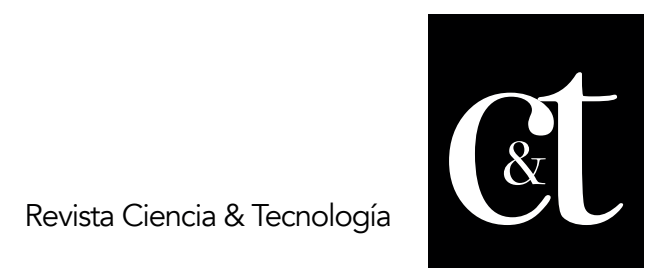

No. 23, 31 de julio de 2019

ISSN impreso: 1390 - 6321

ISSN online: 2661 - 6734

\begin{abstract}
The study of the different methods of cultivation of white shrimp (Litopenaeus vannamei) in low salinity waters, either from underground wells or estuaries, is evaluated from the development to the reception of the larva in a size of 350 animals per gram acclimated to low salinities, through a process of acclimatization in the field placing them for a period of 10 days in raceways or small ponds of 100 tons of water that will allow to provide a better development and management taking care of their survival, for this a later transfer to the pre-breeding or pre-fattening pools remaining about 25 days where they reach an adequate weight, being these juveniles for later sowing in the final fattening ponds where the goal is to reach in the shortest possible time an adequate size to market and maintain survival rates until the end of the cycle, This was done in Ecuador, El Oro province, Huaquillas city.
\end{abstract}

Keywords: shrimp, salinity, days of cultivation, survival

\title{
Introducción
}

La industria camaronera en el Ecuador ha sido de gran puntal y crecimiento, se empezó a desarrollar en el Ecuador desde los años sesenta específicamente por el año 1968. En sus inicios se realizó mediante pesca de arrastre por toda la zona costera del Ecuador, siendo esta una actividad que en cierta parte amenazaba la biodiversidad y la vida marina y donde también sus capturas se centraban únicamente en una sola talla del camarón, no dejando así un desarrollo a nivel marino. Esta actividad para los años 70 se industrializó, implementando nuevas herramientas tecnológicas para mejorar sus producciones.

Históricamente, la actividad camaronera ecuatoriana tiene aproximadamente 50 años de respaldo. Gracias a ello, hoy en día existe un trascendental desarrollo técnico-productivo en lo referente al área de cultivo, cosecha, comercialización y exportación del camarón (Schwarz, 2005). Siendo esta una actividad que empezó a generar grandes réditos económicos, ofreciendo mayores plazas y fuentes de trabajo, ha permitido mejorar los ingresos de las zonas marginales costeras que además sufrían déficit alimentario, siendo éste un recurso clave para ellos para mitigar los escasos recursos obtenidos directamente de la pesca en mar abierto.

Desde los inicios de la actividad camaronera en el Ecuador ha existido un gran apoyo de bancos internacionales, en especial el Banco Mundial y el Banco Internacional de Desarrollo, que permitieron que estos cultivos se desarrollen en este país y que sus exportaciones vayan en aumento obteniendo mayores divisas y retribución económica. $A$ medida que esta actividad se fue desarrollando, vino de la mano una expansión en construcciones inicialmente en las zonas costeras, sobre todo en los archipiélagos de la provincia de El Oro, inicialmente en el sector Jambelí.

Este desarrollo, a su vez, vino de la mano con la poca planificación y control por parte del

Aguirre, Maridueña, Ching, Pérez. Estudiar métodos de producción en el cultivo intensivo de camarón blanco (litopenaeus vannamei) en baja salinidad, una opción para familias emprendedoras 




No. 23, 31 de julio de 2019

ISSN impreso: 1390 - 6321

ISSN online: 2661 - 6734

Gobierno, pues en sus inicios la actividad ocasionaba grandes daños al medio ambiente por la tala incontrolable de manglar en los sectores, desplazando en gran medida el hábitat de diferentes especies como son la concha negra y el cangrejo, cambiando así la iniciativa por la cual se empezó la actividad donde se buscaba producir alimento para satisfacer la demanda de alimentación a la población. Por su parte, el incontrolable crecimiento de las construcciones, en su momento, no fue de gran peso; pues con el transcurso del tiempo se implementaron medidas de control a la expansión.

En el año 1999 Ecuador se enfrentó a una crisis a causa de la enfermedad Mancha Blanca que ocasionó que algunos productores cierren sus cultivos o se mantengan a la espera de encontrar alternativas para controlar la enfermedad.

Los lugares donde están ubicadas las fincas camaroneras en las zonas costeras tienen grandes aspectos naturales como son buenos suelos, buena calidad de agua que han permitido que sean catalogados como excelentes lugares para el desarrollo de la acuacultura en cautiverio. Actualmente la producción de camarón en el Ecuador proviene en un $90 \%$ de cautiverio y el $10 \%$ restante aun lo siguen realizando en altamar pero en menor escala, ya que nuestro camarón en cautiverio ha alcanzado grandes estándares de calidad y por su buen manejo en finca es reconocido a nivel mundial por su sabor y apariencia. En la actualidad el Ecuador en el sector acuícola está muy bien posicionado en la producción de camarón, ubicándose entre las principales actividades económicas del país.

Esta investigación se basa en la producción intensiva de camarón blanco tierra adentro, esto contempla una siembra de las larvas traídas desde sus nauplieras ubicadas en el sector de la península de Santa Elena, Mar Bravo donde pasan por un proceso de desarrollo después de su eclosión de los huevos de las camarones, empezando sus estadios de nauplius que contempla 5 etapas, Protozoe en 3 etapas, Mysis 3 estadios y postlarvas donde les brindan un cuidado en alimentación y temperatura de la mano con una buena calidad de agua y manejo. En este lapso se realiza la aclimatación de la larva ya que desde sus primeros estadios ellas están en un ambiente salino que oscila entre las 25 a 30 ppm de salinidad. Estas son especies que pueden vivir en agua salada y dulce, para los cultivos del estudio que son a base de pozos de agua dulce, las larvas pasan por el proceso de aclimatación en el transcurso de 5 días bajando a 2ppm de salinidad, lo que permite tener una buena salud del animal. Por ende, este estudio va permitir brindar una nueva actividad económica para lugares marginados y alejados a las zonas costeras donde existen comunidades y familias que fácilmente podrían manejar pequeñas extensiones de terrenos, pues siendo estas zonas totalmente áridas arcillosas sería difícil hacer agricultura por la estructura de los suelos; es un emprendimiento que se puede llevar a cabo con ayuda del gobierno o la empresa privada, pues las inversiones no son grandes con la implementación de piscinas pequeñas que vayan desde los tres mil metros a cinco mil metros cuadrados, donde una familia podría manejar de una a dos hectáreas de cultivo tomando en cuenta las condiciones climáticas en temperaturas altas o a su vez la construcción de invernaderos techos que permitirán realizar el cultivo todo el año ya que se podría controlar la temperatura, teniendo un buen 


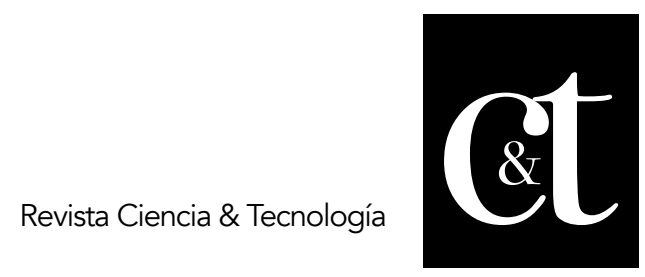

No. 23, 31 de julio de 2019

ISSN impreso: 1390 - 6321

ISSN online: 2661 - 6734

caudal de agua de pozo subterráneo la limitante sería el manejo del balance iónico de estas aguas ya que entre los principales elementos que debe contar el cultivo están el sodio, magnesio y potasio que la fuente de agua no los posee, para lo cual se debe suministrarlo con fuentes inorgánicas. Estos cultivos permitirán mejorar las condiciones de vida de las familias ecuatorianas.

\section{Desarrollo}

El estudio de la crianza de camarón blanco en baja salinidad para sectores marginales que se los cataloga como emprendimientos para comunidades y familias, que va a permitir mejorar su estilo de vida, sus ingresos y fuentes alimenticias, se lo realiza en extensiones pequeñas en piscinas de media hectárea donde una familia puede manejar de una a dos hectáreas entre 2 a 4 piscinas. Esto va a permitir reducir sus costos de producción y tener ventaja al momento de las ventas.

Grupos de pequeñas familias podrían manejarlo con una capacitación base. Este método de producción se puede implementar en zonas que tengan las condiciones apropiadas ya sean estas de temperatura, aunque podría ser controlado a futuro con la construcción de invernaderos techados de plástico para poder regular la temperatura ideal en el cultivo, la cantidad suficiente de agua de pozo subterránea que tenga una salinidad de 1 hasta 5 partes de salinidad. Se pueden implementar en áreas en que los suelos no son aptos para la agricultura pues deben ser netamente arcillosos, estos estanques podrían a su vez servir para cultivar peces que se consuman en la zona o ampliar la gama de productos a ofrecer para tener un ingreso extra.

Estos cultivos en agua dulce están condicionados por numerosas variables, pero en particular se requieren fuentes de aguas con adecuados perfiles iónicos y un manejo correcto de las técnicas de aclimatación de las Pls. Al definir las condiciones para el establecimiento de este tipo de cultivo alternativo se puede sentar las bases para obtener la diversificación de la producción agropecuaria en la región. Además, permite la ocupación de terrenos no aptos para otras actividades agropecuarias por pequeños-medianos productores de camarones en la región (Miranda, Ismael, Valles, José Luis, Sánchez, Roselena, \& Álvarez, Zoraya. 2010).

Para un buen manejo en cautiverio se realiza en los estanques antes mencionados siendo estos de un área no más de media hectárea que son fácilmente manejables, estos son construidos a nivel del suelo con maquinaria agrícola, tractores de gran capacidad que permitan la construcción de muros y excavadoras para perfilar y poder construir los canales de evacuación de aguas para la pesca así mismo una puerta de cosecha. En el centro de las piscinas se debe construir un sifón de un metro de profundidad, quince metros de diámetro instalado con una tubería al exterior con una llave de desfogue que permitirá controlar la evacuación de los sedimentos que se acumulen en el transcurso del cultivo.

Para empezar con una excelente siembra deben los productores tomar en cuenta 


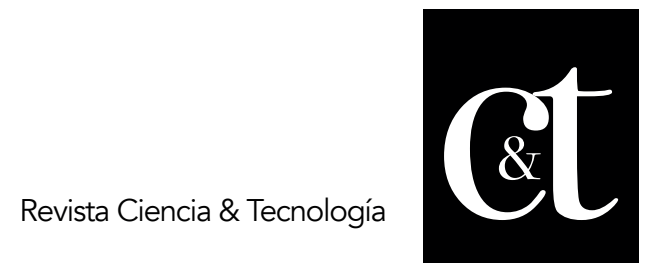

No. 23, 31 de julio de 2019

ISSN impreso: 1390 - 6321

ISSN online: 2661 - 6734

los siguientes parámetros importantes: lo más importante en el cultivo para iniciar es adquirir larvas de excelente calidad que sean desarrolladas con buenas prácticas de manejo y muy bien alimentadas desde el laboratorio. Esto va a permitir que sean resistentes a diferentes patógenos y que a su vez alcancen un buen desarrollo en su crecimiento, suministrándole su alimento balanceado adecuado y cantidad necesaria que va depender del estadio en que se encuentre. Una buena larva siempre va a depender de una buena selección de los reproductores que lo realicen en laboratorio, al respecto existen laboratorios certificados en el Ecuador que nos brindan confiabilidad para la adquisición de una buena larva que es la parte principal para iniciar el cultivo.

Existen sitios en el Ecuador en que las temperaturas no son constantes todo el año por eso para un diseño de estos cultivos de baja salinidad siempre se debe tomar en cuenta la época del año y en temporada de verano las complicaciones por frio no permiten cultivar, es por eso que se debe implementar invernaderos techados para poder controlar y tener un mejor monitoreo de la temperatura ideal para el cultivo de camarón que tiene que ser entre los 28 a 31 grados centígrados.

Al momento de la construcción de las piscinas de engorde se recomienda que tengan una leve inclinación hacia la compuerta de pesca para una fácil evacuación de los camarones, al igual que el sifón en el centro de la piscina para controlar los desechos excedentes de la producción y poder expulsarlos de forma mecánica o manual mediante una bomba de solidos o llaves en el exterior del estanque. Un aspecto muy importante es el suelo que deben ser suelos arcillosos que retengan el agua y no permitan la filtración y sean estos suelos no aptos para la agricultura. Se requiere de equipos aireadores que permitan oxigenar el agua y mantener niveles adecuados para tener buena sobrevivencia, la cantidad de los equipos va a depender de la densidad de siembra y el nivel de agua de los estanques.

Las familias podrían emprender con el manejo adecuado, adquiriendo cada una de estas prácticas mediante capacitaciones abordadas por personal capacitado. Esto permitiría bajar los costos de mano de obra, así también ser más competitivos al momento de su negociación para la venta, el mercado del camarón tiene excelente acogida para la exportación por su peculiaridad de sabor y su renombre que ha adquirido a nivel internacional.

Las prácticas más importantes para un buen manejo es el control de los diferentes parámetros ya sean estos de oxígeno, temperatura, $\mathrm{pH}$ y parámetros químicos en el agua como calcio, magnesio y potasio, que son fundamentales en los cultivos de baja salinidad. Esto se puede hacer mediante equipos digitales o equipos con colorimetría que permiten realizarlos con facilidad en campo y con personal que este muy bien capacitado que pueda interpretar los resultados presentados. Cada uno de estos equipos es de fácil manejo y se pueden adquirir en cualquier distribuidor de insumos y equipos acuícolas.

Se puede implementar un método de siembra en pre criaderos o maternidades donde se acondiciona para la recepción de la larva por un ciclo de 20 a 25 días. Esto va a 


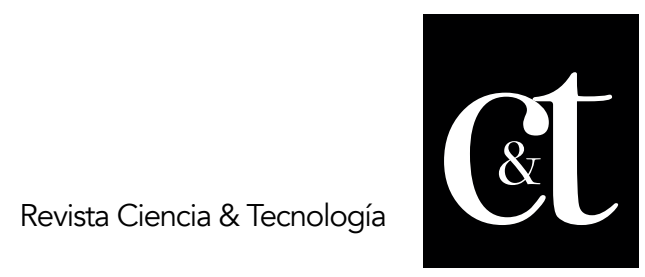

No. 23, 31 de julio de 2019

ISSN impreso: 1390 - 6321

ISSN online: 2661 - 6734

depender del tamaño de la pre-cría, lo que va a permitir adaptar y aclimatar las larvas en sus primeros días y obtener un mejor crecimiento, ya que luego de estos días transcurrido al momento de transferir a piscinas de engorde ganan un crecimiento compensatorio que permite reducir el ciclo de cultivo y por consiguiente un mayor tamaño a la pesca. Estos nuevos métodos de cultivo que se desea implementar en el Ecuador como una alternativa para el desarrollo de las comunidades y familias de escasos recursos, va a permitir un mejor nivel socioeconómico y un mejor aprovechamiento de nuestros recursos naturales o áreas que en la actualidad se encuentran improductivas.

\section{Conclusiones}

El cultivo de camarón blanco es una alternativa para el desarrollo de las zonas marginales y de pocos ingresos en comunidades, donde van a permitir que familias puedan crear pequeñas microempresas, esto debe ir de la mano con la inversión y capacitación por parte del gobierno y apoyo de las entidades bancarias. El Ministerio de Acuacultura y Pesca es el llamado a organizar módulos de capacitación para brindar las diferentes teorías y prácticas que permitan mejorar sus producciones.

Es importante cada una de estas microempresas que se creen en el cultivo de camarón blanco, ya que tendrán una producción considerable en libras que le permita formar clusters para la implementación de una fábrica procesadora y empacadora que cuente con todos los documentos en regla y les permita exportar directamente a mercados extranjeros. "El cultivo de camarón blanco $L$. vannamei es factible en agua subterránea de baja salinidad. En tal sentido, una de las siguientes etapas en las que se debería de poner énfasis, es en el desarrollo de proyectos piloto en zonas de alta marginación, donde a nivel de campo se evalúen las bondades de este tipo de cultivo, a la par de continuar a nivel experimental estudiando el balance iónico óptimo, las tasas de crecimiento y sobrevivencia de la especie. Este estudio proporciona bases para el desarrollo de este tipo de cultivo en zonas marginadas, generando fuentes de empleo en zonas poco desarrolladas." (Valenzuela- Quiñonez, Wenceslao, RodríguezQuiroz, Gerardo, Esparza-Leal, Héctor M).

\section{Referencias bibliográficas}

Miranda, I., Valles, J., Sánchez, R. \& Álvarez, Z. (2010). Cultivo del camarón Liptopenaeus en agua dulce. Revista Científica de la Facultad de Ciencias Veterinarias de la Universidad de Zulia 20(4). Pp. 339-346.

Schwarz, L. (2005). Características, estructura y recursos del sector acuícola del Ecuador. Recuperado de:

http://www.fao.org/fishery/countrysector/naso ecuador/es.

Valenzuela, W., Rodríguez, G., Esparza, H. (2010). Cultivo intensivo de camarón blanco Liptopenaeus (Boone) en agua de pozo de baja salinidad como alternativa acuícola para zonas de alta marginación. Rha Ximhai. Vol. 6 No. 001. 